\title{
On a nonlinear impulsive system of integro-differential equations with degenerate kernel and maxima
}

\author{
Tursun K. Yuldashev ${ }^{1, a}$, Aziz K. Fayziev ${ }^{2, b}$ \\ ${ }^{1}$ National University of Uzbekistan, Tashkent, Uzbekistan \\ ${ }^{2}$ Tashkent State Technical University, Tashkent, Uzbekistan \\ ${ }^{a}$ tursun.k.yuldashev@gmail.com, ${ }^{b}$ fayziyev.a@inbox.ru \\ Corresponding author: Tursun K. Yuldashev, tursun.k.yuldashev@gmail.com
}

ABSTRACT A nonlocal boundary value problem for a system of ordinary integro-differential equations with impulsive effects, degenerate kernel and maxima is investigated. The boundary value problem is given by the integral condition. The method of successive approximations in combination with the method of compressing mapping is used. The existence and uniqueness of the solution of the boundary value problem are proved. The continuous dependence of the solution on the right-hand side of the boundary value condition is shown.

KEYWORDS impulsive integro-differential equations, nonlocal condition, successive approximations, existence and uniqueness, continuous dependence of solution

FOR CITATION Yuldashev T.K., Fayziev A.K. On a nonlinear impulsive system of integro-differential equations with degenerate kernel and maxima. Nanosystems: Phys. Chem. Math., 2022, 13 (1), 36-44.

\section{Introduction}

Many problems in modern sciences, technology and economics are described by differential equations, the solutions of which are functions with first kind discontinuities at fixed or non-fixed times. Such differential equations are called differential equations with impulse effects [1-8]. As is known, in recent years the interest in the study of differential equations with nonlocal boundary conditions has increased (see, for example, [9-17]). In particular, in [15] a physical situation in which a non-metallic conductor is in contact with a perfect conductor is studied. In [16], the problems of mathematical models in reaction-diffusion systems are considered. In [17], the nonlocal conditions are used in the theory of phase transitions.

In [18-24] the problems of solvability for some type of integro-differential equations with degenerate kernel were considered. Also, a lot of publications of studying on differential equations with impulsive effects, describing many natural and technical processes, are appearing [25-35].

In this paper, we investigate a nonlocal boundary value problem for a system of first order Fredholm integrodifferential equations with impulsive effects, degenerate kernel and nonlinear maxima. The questions of the existence and uniqueness of the solution to the boundary value problem, as well as the continuous dependence of the solution on the right-hand side of the boundary condition, are investigated. In [36], it is justified that the theoretical study of differential equations with maxima is relevant.

We consider the following system of Fredholm integro-differential equations:

$$
x^{\prime}(t)=\lambda \int_{0}^{T} H(t, s) x(s) d s+f\left(t, x(t), \max \left\{x(\tau) \mid \tau \in\left[h_{1} ; h_{2}\right]\right\}\right),
$$

for $t \in[0, T], t \neq t_{i}, i=1,2, \ldots, p$ with nonlocal boundary value conditions:

$$
A x(0)+\int_{0}^{T} K(t) x(t) d t=B
$$

and impulsive effect:

$$
x\left(t_{i}^{+}\right)-x\left(t_{i}^{-}\right)=I_{i}\left(x\left(t_{i}\right)\right), \quad i=1,2, \ldots, p,
$$

where $H(t, s)=\sum_{k=1}^{m} a_{k}(t) b_{k}(s), 0=t_{0}<t_{1}<\ldots<t_{p}<t_{p+1}=T, A \in \mathbb{R}^{n \times n}, K(t) \in \mathbb{R}^{n \times n}$ are given matrix and $\operatorname{det} Q \neq 0, Q=A+\int_{0}^{T} K(t) d t, f:[0, T] \times \mathbb{R}^{n} \times \mathbb{R}^{n} \rightarrow \mathbb{R}^{n}, I_{i}: \mathbb{R}^{n} \rightarrow \mathbb{R}^{n}$ are given functions; $0<h_{1}<h_{2}<t$, 
$h_{j}=h_{j}(t, x(t)), j=1,2, \lambda$ is real nonzero parameter, $x\left(t_{i}^{+}\right)=\lim _{h \rightarrow 0^{+}}\left(x_{i}+h\right), x\left(t_{i}^{-}\right)=\lim _{h \rightarrow 0^{-}}\left(t_{i}-h\right)$ are rightsided and left-sided limits of function $x(t)$ at the point $t=t_{i}$, respectively. Every system of functions $\left\{a_{k}(t)\right\}_{k=1}^{m}$ and $\left\{b_{k}(s)\right\}_{k=1}^{m}$ are linearly independent.

\section{Reduction to an integral equation}

Here are some notations that will be used below. We denote by $C\left([0, T], \mathbb{R}^{n}\right)$ the Banach space, which consists of continuous functions $x(t) \in \mathbb{R}^{n}$ on the segment $[0, T]$ with the norm:

$$
\|x\|=\sqrt{\sum_{j=1}^{n} \max _{t \in[0, T]}\left|x_{j}(t)\right|}
$$

Since we consider the integro-differential equation (1) with impulsive effect at the points $t_{i}, i=1,2, \ldots, p$, use the following linear space:

$$
P C\left([0, T], \mathbb{R}^{n}\right)=\left\{x:[0, T] \rightarrow \mathbb{R}^{n} ; x(t) \in C\left(\left(t_{i}, t_{i+1}\right], \mathbb{R}^{n}\right), i=1, \ldots, p\right\},
$$

where $x\left(t_{i}^{+}\right)$and $x\left(t_{i}^{-}\right)(i=0,1, \ldots, p)$ exist and bounded; $x\left(t_{i}^{-}\right)=x\left(t_{i}\right)$.

It is obvious, that the linear space $P C\left([0, T], \mathbb{R}^{n}\right)$ is Banach space with the following norm:

$$
\|x\|_{P C}=\max \left\{\|x\|_{C\left(\left(t_{i}, t_{i+1}\right]\right)}, \quad i=1,2, \ldots, p\right\} .
$$

Formulation of problem. To find the function $x(t) \in P C\left([0, T], \mathbb{R}^{n}\right)$, which for all $t \in[0, T], t \neq t_{i}, i=1,2, \ldots, p$ satisfies the integro-differential equation (1), nonlocal integral condition (2) and for $t=t_{i}, i=1,2, \ldots, p, 0<t_{1}<t_{2}<$ $\ldots<t_{p}<T$ satisfies the limit condition (3).

Let the function $x(t) \in P C\left([0, T], \mathbb{R}^{n}\right)$ is a solution of the problem (1)-(3). Then we rewrite the Fredholm integrodifferential equation (1) as:

$$
x^{\prime}(t)=\lambda \int_{0}^{T} \sum_{k=1}^{m} a_{k}(t) b_{k}(s) x(s) d s+f\left(t, x(t), \max \left\{x(\tau) \mid \tau \in\left[h_{1} ; h_{2}\right]\right\}\right) .
$$

By the designation:

$$
c_{k}=\int_{0}^{T} b_{k}(s) x(s) d s
$$

the last integro-differential equation we rewrite in the following form:

$$
x^{\prime}(t)=\lambda \sum_{k=1}^{m} a_{k}(t) c_{k}+f\left(t, x(t), \max \left\{x(\tau) \mid \tau \in\left[h_{1} ; h_{2}\right]\right\}\right) .
$$

Then, by integration of the last equation on the interval $t \in\left(0, t_{i+1}\right]$, we obtain:

$$
\begin{gathered}
\int_{0}^{t}\left[\lambda \sum_{k=1}^{m} a_{k}(s) c_{k}+f(s, x(s), \cdot)\right] d s= \\
=\int_{0}^{t} x^{\prime}(s) d s=\left[x\left(t_{1}\right)-x\left(0^{+}\right)\right]+\left[x\left(t_{2}\right)-x\left(t_{1}^{+}\right)\right]+\ldots+\left[x(t)-x\left(t_{i}^{+}\right)\right]= \\
=-x(0)-\left[x\left(t_{1}^{+}\right)-x\left(t_{1}\right)\right]-\left[x\left(t_{2}^{+}\right)-x\left(t_{2}\right)\right]-\ldots-\left[x\left(t_{i}^{+}\right)-x\left(t_{i}\right)\right]+x(t) .
\end{gathered}
$$

Taking into account the integral condition (2) in the last equality, we obtain:

$$
x(t)=x(0)+\int_{0}^{t}\left[\lambda \sum_{k=1}^{m} a_{k}(s) c_{k}+f(s, x(s), \cdot)\right] d s+\sum_{0<t_{i}<t} I_{i}\left(x\left(t_{i}\right)\right) .
$$


Let the function $x(t) \in P C\left([0, T], \mathbb{R}^{n}\right)$ in (4), satisfies the boundary value conditions (2):

$$
\begin{aligned}
{\left[A+\int_{0}^{T} K(t) d t\right] x(0) } & = \\
& =B-\int_{0}^{T} K(t) \int_{0}^{t}\left[\lambda \sum_{k=1}^{m} a_{k}(s) c_{k}+f(s, x(s), \cdot)\right] d s d t-\int_{0}^{T} K(t) \sum_{0<t_{i}<t} I_{i}\left(x\left(t_{i}\right)\right) d t .
\end{aligned}
$$

Since $\operatorname{det} Q \neq 0$, from the equality (5) we have:

$$
x(0)=Q^{-1}\left[B-\int_{0}^{T} K(t) \int_{0}^{t}\left[\lambda \sum_{k=1}^{m} a_{k}(s) c_{k}+f(s, x(s), \cdot)\right] d s d t-\int_{0}^{T} K(t) \sum_{0<t_{i}<t} I_{i}\left(x\left(t_{i}\right)\right) d t\right] .
$$

Substituting the equality (6) into representation (4), we obtain:

$$
\begin{aligned}
x(t)=Q^{-1}\left[B-\int_{0}^{T} K(t) \int_{0}^{t}\left[\lambda \sum_{k=1}^{m} a_{k}(s) c_{k}+f(s, x(s), \cdot)\right] d s d t\right. & \left.-\int_{0}^{T} K(t) \sum_{0<t_{i}<t} d t\right]+ \\
& +\int_{0}^{t} f(s, x(s), \cdot) d s+\sum_{0<t_{i}<t} I_{i}\left(x\left(t_{i}\right)\right) .
\end{aligned}
$$

Since the following equalities hold:

$$
\begin{aligned}
\int_{0}^{T} K(t) \int_{0}^{t}\left[\lambda \sum_{k=1}^{m} a_{k}(s) c_{k}+f(s, x(s), \cdot)\right] d s d t & =\int_{0}^{T} \int_{t}^{T} K(s) d s\left[\lambda \sum_{k=1}^{m} a_{k}(t) c_{k}+f(t, x(t), \cdot)\right] d t \\
\int_{0}^{T} K(t) \sum_{0<t_{i}<t} I_{i}\left(x\left(t_{i}\right)\right) d t & =\sum_{0<t_{i}<T} \int_{t_{i}}^{T} K(t) d t I_{i}\left(x\left(t_{i}\right)\right)
\end{aligned}
$$

from presentation (7) we obtain:

$$
\begin{aligned}
x(t)=Q^{-1} B- & Q^{-1} \int_{0}^{T} \int_{t}^{T} K(s) d s\left[\lambda \sum_{k=1}^{m} a_{k}(t) c_{k}+f(t, x(t), \cdot)\right] d t- \\
& -Q^{-1} \sum_{0<t_{i}<t} \int_{t_{i}}^{T} K(t) d t I_{i}\left(x\left(t_{i}\right)\right)+\int_{0}^{t}\left[\lambda \sum_{k=1}^{m} a_{k}(s) c_{k}+f(s, x(s), \cdot)\right] d s+\sum_{0<t_{i}<t} I_{i}\left(x\left(t_{i}\right)\right) .
\end{aligned}
$$

Let us make some simplifications in representation (8). Then the following equalities hold:

$$
\begin{aligned}
& \int_{0}^{t}\left[\lambda \sum_{k=1}^{m} a_{k}(s) c_{k}+f(s, x(s), \cdot)\right] d s-Q^{-1} \int_{0}^{T} \int_{t}^{T} K(s) d s\left[\lambda \sum_{k=1}^{m} a_{k}(t) c_{k}+f(t, x(t), \cdot)\right] d t= \\
& =Q^{-1} \int_{0}^{t}\left(A+\int_{0}^{\theta} K(s) d s\right)\left[\lambda \sum_{k=1}^{m} a_{k}(\theta) c_{k}+f(\theta, x(\theta), \cdot)\right] d \theta- \\
& -Q^{-1} \int_{0}^{T} \int_{t}^{T} K(s) d s\left[\lambda \sum_{k=1}^{m} a_{k}(\theta) c_{k}+f(\theta, x(\theta), \cdot)\right] d \theta \\
& \sum_{0<t_{i}<t} I_{i}\left(x\left(t_{i}\right)\right)-Q^{-1} \sum_{0<t_{i}<T} \int_{t_{i}}^{T} K(t) d t I_{i}\left(x\left(t_{i}\right)\right)= \\
& =Q^{-1} \sum_{0<t_{i}<t}\left(A+\int_{0}^{t_{i}} K(t) d t\right) I_{i}\left(x\left(t_{i}\right)\right)-\sum_{t<t_{i+1}<T} Q^{-1} \int_{t_{i}}^{T} K(t) d t I_{i}\left(x\left(t_{i}\right)\right) .
\end{aligned}
$$


Taking into account (9) and (10), from the presentation (8) we obtain the following integral equation:

$$
x(t)=Q^{-1} B+\sum_{0<t_{i}<t} G\left(t_{i}\right) I_{i}\left(x\left(t_{i}\right)\right)+\lambda \sum_{k=1}^{m} \int_{0}^{T} G(s) a_{k}(s) c_{k} d s+\int_{0}^{T} G(t, s) f(s, x(s), \cdot) d s,
$$

for $t \in\left(t_{i}, t_{i+1}\right], i=0,1, \ldots, p$, where:

$$
G(t)= \begin{cases}Q^{-1}\left(A+\int_{0}^{t} K(s) d s\right), & 0 \leq s \leq t \\ -Q^{-1} \int_{t}^{T} K(s) d s, & t<s \leq T .\end{cases}
$$

Substituting the equation (11) into designation:

$$
c_{k}=\int_{0}^{T} b_{k}(s) x(s) d s
$$

we obtain the following linear system of algebraic equations (LSAE):

$$
c_{k}+\lambda \sum_{j=1}^{m} c_{k j} \Phi_{k j}=\Psi_{1 k}+\Psi_{2 k}\left(f, I_{i}\right), \quad k=\overline{1, m}
$$

where:

$$
\begin{gathered}
\Phi_{k j}\left(f, I_{i}\right)=\int_{0}^{T} b_{k}(s) \int_{0}^{T} G(\theta) a_{j}(\theta) d \theta d s, \quad \Psi_{1 k}=Q^{-1} B \int_{0}^{T} b_{k}(s) d s, \\
\Psi_{2 k}\left(f, I_{i}\right)=\int_{0}^{T} b_{k}(s)\left[\int_{0}^{T} G(\theta) f\left(\theta, x(\theta), \max \left\{x(\tau) \mid \tau \in\left[h_{1} ; h_{2}\right]\right\}\right) d \theta+\sum_{0<t_{i}<t} G\left(t_{i}\right) I_{i}\left(x\left(t_{i}\right)\right)\right] d s, \\
k=\overline{1, m}, h_{l}=h_{l}(t, x(\theta)), l=1,2 .
\end{gathered}
$$

The LSAE (12) is uniquely solvable for any finite right-hand side, if the following Fredholm condition is satisfied:

$$
\Delta_{k}(\lambda)=\left|\begin{array}{cccc}
1+\lambda \Phi_{k 11} & \lambda \Phi_{k 12} & \cdots & \lambda \Phi_{k 1 m} \\
\lambda \Phi_{k 21} & 1+\lambda \Phi_{k 22} & \ldots & \lambda \Phi_{k 2 m} \\
\ldots & \ldots & \ldots & \ldots \\
\lambda \Phi_{k m 1} & \lambda \Phi_{k m 2} & \ldots & 1+\lambda \Phi_{k m m}
\end{array}\right| \neq 0 .
$$
obtain:

Consider such regular values of parameter $\lambda$, for which condition (14) is satisfied. Then, solving LSAE (12), we

where:

$$
c_{k}=\frac{\Delta_{1 k}(\lambda)}{\Delta(\lambda)}+\frac{\Delta_{2 k}\left(\lambda, f, I_{i}\right)}{\Delta(\lambda)}
$$

$$
\Delta_{l k}(\lambda)=\left|\begin{array}{ccccccc}
1+\lambda \Phi_{11} & \ldots & \lambda \Phi_{1(i-1)} & \Psi_{l 1} & \lambda \Phi_{1(i+1)} & \ldots & \lambda \Phi_{1 m} \\
\lambda \Phi_{21} & \ldots & \lambda \Phi_{2(i-1)} & \Psi_{l 2} & \lambda \Phi_{2(i+1)} & \ldots & \lambda \Phi_{2 m} \\
\ldots & \ldots & \ldots & \ldots & \ldots & \ldots & \ldots \\
\lambda \Phi_{m 1} & \ldots & \lambda \Phi_{m(i-1)} & \Psi_{l m} & \lambda \Phi_{m(i+1)} & \ldots & 1+\lambda \Phi_{m m}
\end{array}\right|, \quad l=1,2 .
$$

Substituting equality (15) into representation (11), we obtain the following new presentation of solution:

$$
\begin{aligned}
x(t)=\Theta(t ; x) \equiv \chi_{0}+\lambda \sum_{k=1}^{m}\left[\frac{\Delta_{1 k}(\lambda)}{\Delta(\lambda)}+\frac{\Delta_{2 k}\left(\lambda, f, I_{i}\right)}{\Delta(\lambda)}\right] \chi_{1 k}+ \\
\quad+\int_{0}^{T} G(s) f\left(s, x(s), \max \left\{x(\tau) \mid \tau \in\left[h_{1} ; h_{2}\right]\right\}\right) d s+\sum_{0<t_{i}<t} G\left(t_{i}\right) I_{i}\left(x\left(t_{i}\right)\right),
\end{aligned}
$$


where:

$$
\chi_{0}=Q^{-1} B, \quad \chi_{1 k}=\int_{0}^{T} G(s) a_{k}(s) d s, \quad k=\overline{1, m}, h_{l}=h_{l}(t, x(s)), l=1,2 .
$$

\section{The questions of one value solvability}

Theorem. Suppose the following conditions are fulfilled:

1) For all $t \in[0, T], x, y \in \mathbb{R}^{n}$ holds:

$$
\left|f\left(t, x_{1}, y_{1}\right)-f\left(t, x_{2}, y_{2}\right)\right| \leq M_{1}(t)\left|x_{1}-x_{2}\right|+M_{2}(t)\left|y_{1}-y_{2}\right| ;
$$

2) For all $t \in[0, T], x \in \mathbb{R}^{n}$ holds:

$$
\left|h_{j}\left(t, x_{1}\right)-h_{j}\left(t, x_{2}\right)\right| \leq M_{3 j}(t)\left|x_{1}-x_{2}\right|, j=1,2 ;
$$

3) For all $x \in \mathbb{R}^{n}, i=0,1, \ldots, p$ holds:

$$
\left|I_{i}\left(x_{1}\right)-I_{i}\left(x_{2}\right)\right| \leq m_{i}\left|x_{1}-x_{2}\right|
$$

4) $\rho=S_{1}+S_{2}<1$, where:

$$
\begin{gathered}
S_{1}=|\lambda| \sum_{k=1}^{m}\left|\chi_{1 k}\right| \cdot\left|\bar{\Delta}_{2 k}(\lambda)\right| \int_{0}^{T}|G(s)|\left[M_{1}(s)+M_{2}(s)\left(1+M_{f}\left(M_{31}(s)+M_{32}(s)\right)\right)\right] d s, \\
S_{2}=|\lambda| \sum_{k=1}^{m}\left|\chi_{1 k}\right| \cdot\left|\bar{\Delta}_{2 k}(\lambda)\right| \sum_{i=1}^{p}\left|G\left(t_{i}\right)\right| m_{i}, \\
\bar{\Delta}_{2 k}(\lambda)=\left|\begin{array}{ccccccc}
1+\lambda \Phi_{11} & \ldots & \lambda \Phi_{1(i-1)} & \bar{\Psi}_{21} & \lambda \Phi_{1(i+1)} & \ldots & \lambda \Phi_{1 m} \\
\lambda \Phi_{21} & \ldots & \lambda \Phi_{2(i-1)} & \bar{\Psi}_{22} & \lambda \Phi_{2(i+1)} & \ldots & \lambda \Phi_{2 m} \\
\ldots & \ldots & \ldots & \ldots & \ldots & \ldots & \ldots \\
\lambda \Phi_{m 1} & \ldots & \lambda \Phi_{m(i-1)} & \bar{\Psi}_{2 m} & \lambda \Phi_{m(i+1)} & \ldots & 1+\lambda \Phi_{m m}
\end{array}\right|, \\
\multicolumn{1}{c}{\bar{\Psi}_{2 k}=\int_{0}^{T} b_{k}(s) d s .} \\
0
\end{gathered}
$$

Then, the nonlocal boundary value problem (1)-(3) has a unique solution $x(t) \in P C\left([0, T], \mathbb{R}^{n}\right)$ for the regular values of parameter $\lambda$. This solution can be found from the following iterative process:

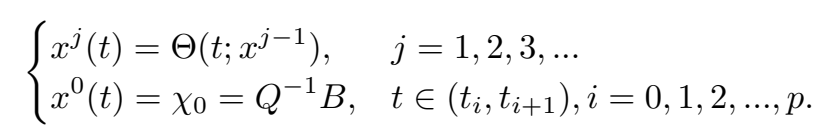

Moreover, for this solution the following estimate is true:

$$
\left\|x_{1}(t)-x_{2}(t)\right\|_{P C} \leq(1-\rho)^{-1}\left\|Q^{-1}\right\| \cdot\left\|B_{1}-B_{2}\right\| .
$$

Proof. We consider the following operator:

$$
\Theta: P C\left([0, T] ; \mathbb{R}^{n}\right) \rightarrow P C\left([0, T] \times \mathbb{R}^{n}\right),
$$

defined by the right-hand side of integral equation (11). Obviously, the fixed point of the operator $\Theta$ is the unique solution to the boundary value problem (1)-(3). Using the principle of contracting operators, we show that the operator $\Theta$ defined by equation (17), has a unique fixed point.

For the zero approximation from (18) we easily obtain that:

$$
\left\|x^{0}(t)\right\| \leq\left\|Q^{-1} B\right\|<\infty .
$$


For the first difference from approximation (18) we have the estimate:

$$
\begin{aligned}
& \left\|x^{1}(t)-x^{0}(t)\right\| \leq|\lambda| \sum_{k=1}^{m}\left[\left|\frac{\Delta_{1 k}(\lambda)}{\Delta(\lambda)}\right|+\left|\frac{\Delta_{2 k}\left(\lambda, f, I_{i}\right)}{\Delta(\lambda)}\right|\right]\left|\chi_{1 k}\right|+ \\
& +\int_{0}^{T}|G(s)| \cdot\left|f\left(s, x^{0}(s), \max \left\{x^{0}(\tau) \mid \tau \in\left[h_{1}^{0} ; h_{2}^{0}\right]\right\}\right)\right| d s+\sum_{i=1}^{p}\left|G\left(t_{i}\right)\right| \cdot\left|I_{i}\left(x^{0}\left(t_{i}\right)\right)\right| \leq \\
& \leq|\lambda| \sum_{k=1}^{m}\left[\left|\frac{\Delta_{1 k}(\lambda)}{\Delta(\lambda)}\right|+\left|\frac{\Delta_{2 k}\left(\lambda, f^{0}, I_{i}^{0}\right)}{\Delta(\lambda)}\right|\right]\left|\chi_{1 k}\right|+S\left(M_{f}+m_{I}\right)<\infty,
\end{aligned}
$$

where:

$$
\begin{gathered}
f^{0}=\int_{0}^{T} G(t) f\left(t, x^{0}(t), \max \left\{x^{0}(\tau) \mid \tau \in\left[h_{1}^{0} ; h_{2}^{0}\right]\right\}\right) d t, \\
I_{i}^{0}=I_{i}\left(x^{0}\left(t_{i}\right)\right), \quad S=\int_{0}^{T}|G(s)| d s+\sum_{i=1}^{p}\left|G\left(t_{i}\right)\right|, \\
M_{f}=\max _{t \in[0, T]}\left|f\left(t, Q^{-1} B, Q^{-1} B\right)\right|, \quad m_{I}=\max _{i \in\{1,2, \ldots, p\}}\left|I_{i}\left(Q^{-1} B\right)\right| .
\end{gathered}
$$

Then, by virtue of the conditions of the theorem and (13), (16), for arbitrary $t \in\left(t_{i}, t_{i+1}\right]$ we have:

$$
\begin{aligned}
& \left|x^{j}(t)-x^{j-1}(t)\right| \leq|\lambda| \sum_{k=1}^{m}\left|\frac{\left|\Delta_{2 k}\left(\lambda, f^{j-1}, I_{i}^{j-1}\right)-\Delta_{2 k}\left(\lambda, f^{j-2}, I_{i}^{j-2}\right)\right|}{\Delta(\lambda)}\right|\left|\chi_{1 k}\right|+ \\
& +\int_{0}^{T}|G(s)| \cdot\left|f\left(s, x^{j-1}(s), \max \left\{x^{j-1}(\tau) \mid \tau \in\left[h_{1}^{j-1} ; h_{2}^{j-1}\right]\right\}\right)-f\left(s, x^{j-2}(s), \max \left\{x^{j-2}(\tau) \mid \tau \in\left[h_{1}^{j-2} ; h_{2}^{j-2}\right]\right\}\right)\right| d s+ \\
& +\sum_{i=1}^{p}\left|G\left(t_{i}\right)\right| \cdot\left|I_{i}\left(x^{j-1}\left(t_{i}\right)\right)-I_{i}\left(x^{j-2}\left(t_{i}\right)\right)\right| \leq \\
& \left.+M_{2}(s) \cdot\left|\max \left\{x_{k=1}^{j-1}(\tau) \mid \tau \in\left[h_{1}^{j-1} ; h_{2}^{j-1}\right]\right\}-\max \left\{x^{j-2}(\tau) \mid \tau \in\left[h_{1}^{j-2} ; h_{2}^{j-2}\right]\right\}\right|\right] d s+ \\
& +|\lambda| \sum_{k=1}^{m}\left|\chi_{1 k}\right| \cdot\left|\bar{\Delta}_{2 k}(\lambda)\right| \cdot \sum_{i=1}^{p}\left|G\left(t_{i}\right)\right| \cdot m_{i} \cdot\left|x^{j-1}\left(t_{i}\right)-x^{j-2}\left(t_{i}\right)\right|+\int_{0}^{T}|G(s)| \cdot\left[M_{1}(s) \cdot\left|x^{j-1}(s)-x^{j-2}(s)\right|+\right. \\
& \left.+M_{2}(s) \cdot\left|\max \left\{x^{j-1}(\tau) \mid \tau \in\left[h_{1}^{j-1} ; h_{2}^{j-1}\right]\right\}-\max \left\{x^{j-2}(\tau) \mid \tau \in\left[h_{1}^{j-2} ; h_{2}^{j-2}\right]\right\}\right|\right] d s+ \\
& +\sum_{i=1}^{p}\left|G\left(t_{i}\right)\right| \cdot m_{i} \cdot\left|x^{j-1}\left(t_{i}\right)-x^{j-2}\left(t_{i}\right)\right|
\end{aligned}
$$

where $h_{l}^{j}=h_{l}\left(t, x^{j}(t)\right), l=1,2$ and:

$$
\bar{\Delta}_{2 k}(\lambda)=\left|\begin{array}{ccccccc}
1+\lambda \Phi_{11} & \ldots & \lambda \Phi_{1(i-1)} & \bar{\Psi}_{21} & \lambda \Phi_{1(i+1)} & \ldots & \lambda \Phi_{1 m} \\
\lambda \Phi_{21} & \ldots & \lambda \Phi_{2(i-1)} & \bar{\Psi}_{22} & \lambda \Phi_{2(i+1)} & \ldots & \lambda \Phi_{2 m} \\
\ldots & \ldots & \ldots & \ldots & \ldots & \ldots & \ldots \\
\lambda \Phi_{m 1} & \ldots & \lambda \Phi_{m(i-1)} & \bar{\Psi}_{2 m} & \lambda \Phi_{m(i+1)} & \ldots & 1+\lambda \Phi_{m m}
\end{array}\right|,
$$


By virtue of third condition of the theorem, we have:

$$
\begin{aligned}
& \mid \max \left\{x^{j-1}(\tau) \mid\right.\left.\in\left[h_{1}^{j-1} ; h_{2}^{j-1}\right]\right\}-\max \left\{x^{j-2}(\tau) \mid \tau \in\left[h_{1}^{j-2} ; h_{2}^{j-2}\right]\right\} \mid \leq \\
& \leq\left|\max \left\{x^{j-1}(\tau) \mid \tau \in\left[h_{1}^{j-1} ; h_{2}^{j-1}\right]\right\}-\max \left\{x^{j-2}(\tau) \mid \tau \in\left[h_{1}^{j-1} ; h_{2}^{j-1}\right]\right\}\right|+ \\
& \quad+\left|\max \left\{x^{j-2}(\tau) \mid \tau \in\left[h_{1}^{j-1} ; h_{2}^{j-1}\right]\right\}-\max \left\{x^{j-2}(\tau) \mid \tau \in\left[h_{1}^{j-2} ; h_{2}^{j-2}\right]\right\}\right| \leq \\
& \leq\left|x^{j-1}(t)-x^{j-2}(t)\right|+M_{f}\left[\left|h_{1}\left(t, x^{j-1}(t)\right)-h_{1}\left(t, x^{j-2}(t)\right)\right|+\left|h_{2}\left(t, x^{j-1}(t)\right)-h_{2}\left(t, x^{j-2}(t)\right)\right|\right] \leq \\
& \leq\left(1+M_{f}\left(M_{31}(t)+M_{32}(t)\right)\right)\left|x^{j-1}(t)-x^{j-2}(t)\right| .
\end{aligned}
$$

Substituting the estimate (22) into (21), we obtain:

where $\rho=S_{1}+S_{2}$ and:

$$
\left\|x^{j}(t)-x^{j-1}(t)\right\|_{P C} \leq \rho \cdot\left\|x^{j-1}(t)-x^{j-2}(t)\right\|_{P C},
$$

$$
\begin{gathered}
S_{1}=|\lambda| \sum_{k=1}^{m}\left|\chi_{1 k}\right| \cdot\left|\bar{\Delta}_{2 k}(\lambda)\right| \int_{0}^{T}|G(s)| \cdot\left[M_{1}(s)+M_{2}(s)\left(1+M_{f}\left(M_{31}(s)+M_{32}(s)\right)\right)\right] d s \\
S_{2}=|\lambda| \sum_{k=1}^{m}\left|\chi_{1 k}\right| \cdot\left|\bar{\Delta}_{2 k}(\lambda)\right| \sum_{i=1}^{p}\left|G\left(t_{i}\right)\right| \cdot m_{i} .
\end{gathered}
$$

According to the last condition of the theorem, $\rho<1$. Therefore, from the estimate (23) we have:

$$
\left\|x^{j}(t)-x^{j-1}(t)\right\|_{P C}<\left\|x^{j-1}(t)-x^{j-2}(t)\right\|_{P C} .
$$

It follows from (24) that the operator $\Theta$ on the right-hand side of (17) is contracting. According to fixed point principle, taking into account estimates (19), (20) and (24), we conclude that the operator $\Theta$ has a unique fixed point. Consequently, the nonlocal boundary value problem (1)-(3) has a unique solution $x(t) \in P C\left([0, T], \mathbb{R}^{n}\right)$.

Now, let us show the continuous dependence of the solution to the boundary value problem (1)-(3) on the right-hand side of condition (2). Let $B_{1}, B_{2} \in \mathbb{R}^{n}$ are two different constants and $x_{1}(t), x_{2}(t) \in P C\left([0, T], \mathbb{R}^{n}\right)$ are corresponding solutions of the problem (1)-(3). Then, we have:

$$
\begin{aligned}
x_{1}(t) & -x_{2}(t)=Q^{-1}\left[B_{1}-B_{2}\right]+\lambda \sum_{k=1}^{m} \frac{\Delta_{2 k}\left(\lambda, f_{1}, I_{1 i}\right)-\Delta_{2 k}\left(\lambda, f_{2}, I_{2 i}\right)}{\Delta(\lambda)} \chi_{1 k}+ \\
& +\int_{0}^{T} G(s) \cdot\left|f\left(s, x_{1}(s), \max \left\{x_{1}(\tau) \mid \tau \in\left[h_{1}^{1} ; h_{2}^{1}\right]\right\}\right)-f\left(s, x_{2}(s), \max \left\{x_{2}(\tau) \mid \tau \in\left[h_{1}^{2} ; h_{2}^{2}\right]\right\}\right)\right| d s+ \\
& +\sum_{i=1}^{P} G\left(t_{i}\right)\left[I_{i}\left(x_{1}\left(t_{i}\right)\right)-I_{i}\left(x_{2}\left(t_{i}\right)\right)\right],
\end{aligned}
$$

where $h_{j}^{k}=h_{j}\left(t, x_{k}(t)\right), j, k=1,2$. Now, using the first two conditions of the theorem, from (25) we obtain:

$$
\begin{aligned}
& \left|x_{1}(t)-x_{2}(t)\right| \leq Q^{-1}\left[B_{1}-B_{2}\right]+|\lambda| \sum_{k=1}^{m}\left|\frac{\left|\Delta_{2 k}\left(\lambda, f_{1}, I_{1 i}\right)-\Delta_{2 k}\left(\lambda, f_{2}, I_{2 i}\right)\right|}{\Delta(\lambda)}\right|\left|\chi_{1 k}\right|+ \\
& +\int_{0}^{T}|G(s)| \cdot\left[M_{1}(s) \cdot\left|x_{1}(s)-x_{2}(s)\right|+M_{2}(s) \cdot\left|\max \left\{x_{1}(\tau) \mid \tau \in\left[h_{1}^{1} ; h_{2}^{1}\right]\right\}-\max \left\{x_{2}(\tau) \mid \tau \in\left[h_{1}^{2} ; h_{2}^{2}\right]\right\}\right|\right] d s+ \\
& +\sum_{i=1}^{p}\left|G\left(t_{i}\right)\right| \cdot m_{i} \cdot\left|x_{1}\left(t_{i}\right)-x_{2}\left(t_{i}\right)\right| .
\end{aligned}
$$

Hence, as in the case of estimation process for (23), we obtain:

$$
\left\|x_{1}(t)-x_{2}(t)\right\|_{P C} \leq\left\|Q^{-1}\right\|\left\|B_{1}-B_{2}\right\|+\rho \cdot\left\|x_{1}(t)-x_{2}(t)\right\|_{P C} .
$$

Since $\rho<1$, from the last inequality, it follows that:

$$
\left\|x_{1}(t)-x_{2}(t)\right\|_{P C} \leq(1-\rho)^{-1}\left\|Q^{-1}\right\| \cdot\left\|B_{1}-B_{2}\right\| .
$$

If we put $\left\|B_{1}-B_{2}\right\|<\delta$ and $\varepsilon=(1-\rho)^{-1}\left\|Q^{-1}\right\| \cdot \delta$, then, from the last inequality, we obtain $\left\|x_{1}(t)-x_{2}(t)\right\|_{P C}<\varepsilon$. The theorem is proved. 


\section{Conclusion}

The theory of differential equations plays an important role in solving applied problems. Especially, nonlocal boundary value problems for differential equations with impulsive actions have many applications in mathematical physics, mechanics and technology, in particular in nanotechnology.

In this paper, we investigated the system of first order Fredholm integro-differential equations (1) with nonlocal boundary value condition (2) and with condition (3) of impulsive effects for $t=t_{i} i=1,2, \ldots, p, 0<t_{1}<t_{2}<\ldots<$ $t_{p}<T$. The kernel of integro-differential equation (1) is degenerate. The nonlinear right-hand side of this equation consists of the construction of nonlinear maxima. The questions of the existence and uniqueness of the solution of the boundary value problem (1)-(3) are studied. The continuous dependence of the solution on the right-hand side of the boundary condition was proved.

The results obtained in this work will allow us in the future to investigate nonlocal boundary value problems for the heat equation and the wave equation with impulsive actions. We hope that our work will stimulate the study of various boundary value problems for partial differential and integro-differential equations with impulsive actions.

\section{References}

[1] Benchohra M., Henderson J., Ntouyas S.K. Impulsive differential equations and inclusions. Contemporary mathematics and its application. Hindawi Publishing Corporation, New York, 2006.

[2] Boichuk A.A., Samoilenko A.M. Generalized inverse operators and Fredholm boundary-value problems. Utrecht, Brill, 2004.

[3] Boichuk A.A., Samoilenko A.M. Generalized inverse operators and Fredholm boundary-value problems (2nd ed.). Walter de Gruyter GmbH, Berlin-Boston, 2016. 314 p.

[4] Islomov B.I., Abdullaev A.A. On a problem for an elliptic type equation of the second kind with a conormal and integral condition. Nanosystems: Physics, Chemistry, Mathematics, 2018, 9 (3), P. 307-318.

[5] Lakshmikantham V., Bainov D.D., Simeonov P.S. Theory of impulsive differential equations. World Scientific, Singapore, 1989. 434 p.

[6] Perestyk N.A., Plotnikov V.A., Samoilenko A.M., Skripnik N.V. Differential equations with impulse effect: multivalued right-hand sides with discontinuities. DeGruyter Stud. 40, Math. Walter de Gruter Co., Berlin, 2011.

[7] Samoilenko A.M., Perestyk N.A. Impulsive differential equations. World Sci., Singapore, 1995.

[8] Halanay A., Veksler D. Qualitative theory of impulsive systems. Mir, Moscow, 1971. 309 p. (in Russian).

[9] Assanova A. An integral-boundary value problem for a partial differential equation of second order. Turkish J. of Mathematics, 2019, 43 (4), P. 1967-1978.

[10] Assanova A.T. On the solvability of nonlocal problem for the system of Sobolev-type differential equations with integral condition. Georgian Mathematical J., 2021, 28 (1), P. 49-57.

[11] Assanova A.T., Imanchiyev A.E., Kadirbayeva Zh.M. A nonlocal problem for loaded partial differential equations of fourth order. Bulletin of the Karaganda university - Mathematics, 2020,97 (1), P. 6-16.

[12] Assanova A.T., Tokmurzin Z.S. A nonlocal multipoint problem for a system of fourth-order partial differential equations. Eurasian Math. J., 2020, 11 (3), P. 8-20.

[13] Minglibayeva A.B., Assanova A.T. An existence of an isolated solution to nonlinear two-point boundary value problem with parameter. Lobachevskii J. of Mathematics, 2021, 42 (3), P. 587-597.

[14] Yuldashev T.K. Nonlinear optimal control of thermal processes in a nonlinear inverse problem. Lobachevskii J. of Mathematics, 2020, 41 (1), P. 124-136.

[15] Gianni R. Equation with nonlocal boundary condition. Mathematical Models and Methods in Applied Sciences, 1993, 3 (6), P. $789-804$.

[16] Pao C.V. Numerical solutions of reaction-diffusion equations with nonlocal boundary conditions. J. of Computational and Applied Mathematics, 2001, 136 (1-2), P. 227-243.

[17] Giacomin G., Lebowitz J.L. Phase segregation dynamics in particle systems with long range interactions, I. Macroscopic limits. J. of Statist. Phys., 1997, 87, P. 37-61.

[18] Yuldashev T.K. Nonlocal mixed-value problem for a Boussinesq-type integrodifferential equation with degenerate kernel. Ukrainian Mathematical J., 2016, 68 (8), P. 1278-1296.

[19] Yuldashev T.K. Determination of the coefficient and boundary regime in boundary value problem for integro-differential equation with degenerate kernel. Lobachevskii J. of Mathematics, 2017, 38 (3), P. 547-553.

[20] Yuldashev T.K. Nonlocal boundary value problem for a nonlinear Fredholm integro-differential equation with degenerate kernel. Differential equations, 2018, 54 (12), P. 1646-1653.

[21] Yuldashev T.K. Spectral features of the solving of a Fredholm homogeneous integro-differential equation with integral conditions and reflecting deviation. Lobachevskii J. of Mathematics, 2019, 40 (12), P. 2116-2123.

[22] Yuldashev T.K. On inverse boundary value problem for a Fredholm integro-differential equation with degenerate kernel and spectral parameter. Lobachevskii J. of Mathematics, 2019, 40 (2), P. 230-239.

[23] Yuldashev T.K. On the solvability of a boundary value problem for the ordinary Fredholm integrodifferential equation with a degenerate kernel. Computational Mathematics and Math. Physics, 2019, 59 (2), P. 241-252.

[24] Yuldashev T.K. On a Volterra type fractional integro-differential equation with degenerate kernel. AIP Conference Proceedings, 2021, 2365, 020016.

[25] Annamalai Anguraj, Mani Mallika Arjunan. Existence and uniqueness of mild and classical solutions of impulsive evolution equations. Elect. J. of Differential Equations, 2005, 2005 (111), P. 1-8.

[26] Ashyralyev A., Sharifov Y.A. Existence and uniqueness of solutions for nonlinear impulsive differential equations with two-point and integral boundary conditions. Advances in Difference Equations, 2013, 2013 (173).

[27] Ashyralyev A., Sharifov Y.A. Optimal control problems for impulsive systems with integral boundary conditions. Elect. J. of Differential Equations, 2013, 2013 (80), P. 1-11.

[28] Bin L., Xinzhi L., Xiaoxin L. Robust global exponential stability of uncertain impulsive systems. Acta Mathematika Scientia, 2005, 25 (1), P. 161169. 
[29] Ji Sh., Wen Sh. Nonlocal cauchy problem for impulsive differential equations in Banach spaces. Intern. J. of Nonlinear Science, 2010, 10 (1), P. 88-95.

[30] Li M., Han M. Existence for neutral impulsive functional differential equations with nonlocal conditions. Indagationes Mathematcae, 2009, 20 (3), P. 435-451.

[31] Mardanov M.J., Sharifov Ya.A., Molaei H.H. Existence and uniqueness of solutions for first-order nonlinear differential equations with two-point and integral boundary conditions. Electr. J. of Differential Equations, 2014, 2014 259), P. 1-8.

[32] Sharifov Ya.A. Optimal control problem for systems with impulsive actions under nonlocal boundary conditions. Vestnik Samarskogo Gosudarstvennogo Tekhnicheskogo Universiteta. Seria: Fiziko-matematicheskie nauki, 2013, 4 (33), P. $34-45$ (in Russian).

[33] Sharifov Ya.A. Optimal control for systems with impulsive actions under nonlocal boundary conditions. Russian Mathematics (Izv. VUZ), 2013, 57 (2), P. 65-72.

[34] Sharifov Y.A., Mammadova N.B. Optimal control problem described by impulsive differential equations with nonlocal boundary conditions. Differential equations, 2014, 50 (3), P. 403-411.

[35] Sharifov Y.A. Conditions optimality in problems control with systems impulsive differential equations with nonlocal boundary conditions. Ukrainain Math. J., 2012, 64 (6), P. 836-847.

[36] Yuldashev T.K. Limit value problem for a system of integro-differential equations with two mixed maxima. Vestnik Samarskogo Gosudarstvennogo Tekhnicheskogo Universiteta. Seria: Fiziko-matematicheskie nauki, 2008, 1 (16), P. 15-22 (in Russian).

Submitted 28 November 2021; revised 26 December 2021; accepted 28 December 2021

Information about the authors:

Tursun K. Yuldashev - National University of Uzbekistan, Universitet street, 4, NUUz, Tashkent, 100174, Uzbekistan; ORCID 0000-0002-9346-5362; tursun.k.yuldashev@gmail.com

Aziz K. Fayziev - Tashkent State Technical University, Universitet street, 2, TSTU, Tashkent, 100174, Uzbekistan; ORCID 0000-0001-6798-3265; fayziyev.a@inbox.ru

Conflict of interest: the authors declare no conflict of interest. 